\title{
Turbulent Length Scale for Multilayer RANS Model of Urban Canopy and Its Evaluation Based on Large-Eddy Simulations
}

\author{
Andrey V. Glazunov ${ }^{1,2,3}$ (D), Andrey V. Debolskiy ${ }^{3,4}$ (iD, \\ Evgeny V. Mortikov ${ }^{1,3}$
}

(C) The Authors 2021. This paper is published with open access at SuperFri.org

\begin{abstract}
Large-Eddy Simulation (LES) numerical experiments of neutrally-stratified turbulent flow over an urban-type surface and passive scalar transport by this flow are performed. A simple parameterization of the turbulent length scale containing only one empirical constant is proposed. Multilayer Reynolds-Averaged Navier-Stokes (RANS) model of turbulent flow and turbulent scalar diffusion is constructed. The results of the RANS model are compared with the LES experiments. It is shown that the proposed approach allows predicting the average flow velocity and the scalar concentration inside and above the urban canopy.

Keywords: atmospheric boundary layer, numerical simulation of turbulence, urban canopy, scalar turbulent transport.
\end{abstract}

\section{Introduction}

Increasing performance of supercomputers makes it possible to make detailed weather forecasts, in which the horizontal scale of large metropolitan areas is resolved explicitly on grids of General Circulation Models (GCMs). One of the important blocks of large-scale models are local one-dimensional RANS models of the atmospheric boundary layer, which describe the near surface turbulent transport of momentum and scalar quantities vertically. In most contemporary GCMs, any surface, including the urban canopy, is considered a rough surface coinciding with the smoothed terrain, and its small-scale geometric features (e.g., high-rise buildings) are taken into account only in terms of their integral impact on the turbulent exchange processes between the surface as a whole and the atmosphere above. At the same time, along with grid refinement of GCMs horizontally, their vertical resolution also increases so that for several grid nodes with a step of the order of several meters close to the surface there are city buildings which induce form drag that needs to be accounted for. So, it is necessary to develop multilayer one-dimensional turbulence models that take into account the dynamic and thermal effects of buildings and vegetation on turbulence in the form of vertically distributed forcing and incorporate special turbulent closures for them. In such models, the surface layer is considered as a porous medium, which creates volumetric resistance to the mean wind and is capable of generating turbulent kinetic energy in the flow around buildings.

The first such models appeared quite a long time ago (see, e.g., [17]) and now incorporate parameterizations of various physical processes, such as multilayer parameterizations of radiation sources and heat sinks, the dynamic and thermal interactions of turbulence with vegetation (see $5,14,16,20]$ ). These models will eventually make it possible to refine the meteorological characteristics within the urban canopy near surface. In addition, an important stimulus for the development of multilayer RANS models is the need to predict the concentrations of pollutants represented by gaseous impurities and particulate matter.

\footnotetext{
${ }^{1}$ G.I. Marchuk Institute of Numerical Mathematics, Russian Academy of Science, Moscow, Russian Federation

${ }^{2}$ Moscow Center for Fundamental and Applied Mathematics, Moscow, Russia

${ }^{3}$ Research Computing Center, Lomonosov Moscow State University, Moscow, Russian Federation

${ }^{4}$ A.M. Obukhov Institute of Atmospheric Physics,Russian Academy of Science, Moscow, Russian Federation
} 
One of the most important problems arising in the development of multilayer onedimensional models of the urban canopy is the difficulty of independent evaluation of their individual components. Measurements in real cities are not always feasible, since they are subject to the combined influence of numerous external meteorological factors and have significant spatial heterogeneity. There is a need for evaluation of RANS models in idealized conditions simulating urban turbulence in a state of statistical equilibrium and spatial homogeneity on horizontal scales exceeding the size of large elements of urban surface roughness. It is possible to create such conditions in two ways. The first one is traditional and consists of setting up specialized laboratory experiments to measure the statistical characteristics of turbulence of a flow around objects similar to buildings, but with simple shape and regular structure of their location on the surface (see, for example, [3]).

For laboratory experiments performing high Reynolds number flow experiments, comparable to urban canopy in atmospheric boundary layer, remains a challenge. Researchers resort to some combined way of organizing measurements, placing large arrays of simple in shape large objects (e.g., cubes of a $1.5 \mathrm{~m}$ size) in the open air, rather than in the laboratory setup (see, experiment COSMO (Comprehensive Outdoor Scale Model) http://www.ide.titech.ac.jp/ kandalab/COSMO/COSMO.html [13]). This approach provided reliable data for developing parameterizations of the dynamic and thermal interaction of turbulence with urban-type surfaces (see, e.g., [12]).

Another way to obtain low noise, detailed and sufficiently reliable data on turbulent dynamics and turbulent scalar transport in urban-type environments is direct numerical simulation (DNS) and large-eddy simulation (LES). The use of numerical models (both LES and DNS) to establish the aerodynamic characteristics of urban-type surfaces is not new. The first such numerical simulations were performed more than 15 years ago [4, 21] and showed good agreement with laboratory data 3]. Contemporary HPC systems, not only allow us to significantly extend the range of surface configurations considered, but also to accurately resolve the characteristics of turbulent flow within the $0<z<h$ layer filled with roughness objects. This, in particular, makes it possible to use LES data to construct and verify multilayer local one-dimensional RANS models.

In 19] the LES data obtained from flow simulations over an array of cubes (see Fig. 3 of [19]), a second order (K-l) multilayer model based on prognostic equation for turbulent kinetic energy (TKE) and prescribed turbulent length scales within the canopy layer was calibrated and validated: $l_{m}$ to calculate the turbulent viscosity and TKE diffusion coefficients and $l_{\varepsilon}$ to calculate TKE dissipation rate. In that study universal functions $\tilde{l}_{m}(\tilde{z})$ and $\tilde{l}_{\varepsilon}(\tilde{z})$ of these scales are proposed, which depend on the geometric parameters of urban canopy (here: $\tilde{l}_{m}=l_{m} / h$, $\tilde{l}_{\varepsilon}=l_{\varepsilon} / h, \tilde{z}=z / h$ are normalized by the thickness of the urban canopy layer). The onedimensional model is constructed by introducing an additional drag force into the equation for the average velocity $U$ :

$$
F_{D}=-C_{D} U|U|
$$

where $C_{D}$ is positive inside the layer $0<z<h$ and, generally speaking, depends on $z$ volume drag coefficient with dimensions $\left[C_{D}\right]=\mathrm{m}^{-1}$. In addition, a new term is added to the TKE balance equation, which describes its production through the interaction with the buildings:

$$
P_{D}=C_{D}|U|^{3}
$$


The authors of $[19]$ have shown with their tests that the parameterization calibrated with LES data improves the reproduction of the average velocity $U$ both inside and above the $0<z<h$ layer. In addition, that paper has provided comparisons with other independent simulations and measurements.

Our study has two research objectives. First, we will try to test the performance of the proposed parameterizations for surfaces with relatively small object densities (on the grounds that when including them in GCMs, we will first have to consider the impact of the highest buildings, which are typically represented with a small fraction). Second, we will test how the proposed parameterizations model turbulent scalar transport within canopy. These goals seem to us even more relevant than modelling of the mean wind and its dispersion profiles in the urban environment, because it is directly related to the possibility of forecast of pollutants concentrations.

In addition, we propose the simplest way to construct a turbulent length scale within the roughness layer. This scale is derived from dimensional considerations and includes only one empirical parameter. In addition, we propose to calculate this scale without using data of the geometry of the urban surface, which is extremely difficult to generalize and express by a single number. We suggest that the nature of the turbulence itself can do this generalization for us. Indeed, above the roughness layer at large Reynolds numbers and for neutral stratification, the profile of the dimensionless mean wind velocity $\tilde{U}$ can be approximated by a logarithmic relationship:

$$
\tilde{U}=\frac{U}{U_{*}}=\frac{1}{\kappa} \ln \left(\frac{z-D_{u}}{z_{0 u}}\right),
$$

where $U_{*}$ is the friction velocity $\left(U_{*}=\left|\tau_{s}\right|^{1 / 2} ; \tau_{s}\right.$ is the mean tangential frictional stress on the surface as a whole normalized by air density); $\kappa \approx 0.4$ is the von Karman constant; $z_{0 u}$ is the roughness length parameter, $D_{u}$ is the displacement height. The parameter $z_{0 u} \ll h$ has the meaning of a height independent additive constant to the entire dimensionless velocity profile and characterizes the surface as a whole in terms of the efficiency of momentum exchange with the atmosphere, and hence it is, to a greater extent, related to the value of the drag coefficient $C_{D}$. At the same time, the validity of using the height $z^{\prime}=\left(z-D_{u}\right)$ in the approximation 3 suggests that the characteristic turbulent length scales in the flow over the complex surface due to the influence of surface geometry are proportional to the scale $z^{\prime}=z-D_{u}$ (instead of their proportionality to the scale $z$ over the flat wall) with small corrections. Our null hypothesis is that the dimensionless parameter $D_{u} / h$ characterizes the turbulent length scales both above and inside the canopy layer, since at least the largest turbulent fluctuations obviously cannot be independent outside and inside the urban environment. The specific form of our proposed approximation is given below. Note that the parameters $z_{0 u}$ and $D_{u}$ are measurable and can be obtained from meteorological observations and eddy covariance momentum flux measurements over the urban surface.

Below we present the results of numerical experiments with the INM RAS LES model 77, which has been previously repeatedly tested for modeling turbulence over urban surfaces 8 [11]. The experiments have been performed in the traditional formulation, which coincides, except for minor details, with the numerical experiments design carried out in [4, 21] and [19]. In addition to modeling the flow itself, we calculated the turbulent scalar transport with the source at the surface.

A one-equation $K-l$ turbulence model similar to the [19] model was used as a local onedimensional RANS model and, in the same way, augmented with parameterization of the bulk 
form drag (1) and TKE production 22. The main difference is in the choice of the turbulent length scale. For comparison, we also present the results of calculations with our RANS model with the length scales proposed in 19]. Here we do not investigate the parameterization of the $C_{D}$ coefficient, so we will use the coefficient calculated from LES data for its assignment in both RANS models.

The results of the local-one-dimensional models are compared with the LES data and with each other. We will show that our proposed simple parameterization of the turbulent scale on the considered surface geometries is not inferior, and in some cases superior, to the parameterizations built on the basis of generalization of geometric parameters of the urban environment.

The article is organized as follows. The sections 1 and 2 provide a brief descriptions of the models (LES and RANS, correspondingly). Section 3 describes the setup of numerical experiments with the models and the parameters of numerical calculations. The proposed turbulent length scale is presented in section 5 . Section 6 shows the results of RANS calculations and their comparison with LES data.

\section{LES Model Description}

The model explicitly reproduces the filtered velocity $\overline{\mathbf{u}} \equiv F_{\bar{\Delta}}(\mathbf{u})$, except for small-scale fluctuations $\mathbf{u}^{\prime \prime}=\mathbf{u}-\overline{\mathbf{u}}$ (here: $F_{\bar{\Delta}}$ is a given spatial filter commuting with differentiation operators). The differential equations for conservation of momentum and mass of incompressible fluid in tensor notation have the following form:

$$
\frac{\partial \bar{u}_{i}}{\partial t}+\frac{\partial \bar{u}_{i} \bar{u}_{j}}{\partial x_{j}}=-\frac{\partial \tau_{i j}}{\partial x_{j}}-\frac{\partial \bar{p}}{\partial x_{i}}+\overline{F_{i}^{e}}, \quad \frac{\partial \bar{u}_{i}}{\partial x_{i}}=0,
$$

where $F_{i}^{e}$ corresponds to the external force acting on the flow, as well as the Coriolis acceleration and buoyancy forces (equal to zero in the case under consideration); $\bar{p}$ is the normalized pressure; $\tau_{i j}=\overline{u_{i} u_{j}}-\overline{u_{i}} \overline{u_{j}}$ is the "sub-grid/ sub-filter" stress tensor, subject to parameterization. The term involving the kinematic viscosity of air $\nu$ is usually neglected in LES models of atmospheric boundary layer flows.

The system of equations 4 is supplemented by the filtered scalar transport equation $\bar{s}$ :

$$
\frac{\partial \bar{s}}{\partial t}+\bar{u}_{i} \frac{\partial \bar{s}}{\partial x_{i}}=-\frac{\partial \vartheta_{i}^{s}}{\partial x_{i}}+\overline{F_{s}}
$$

where $\overline{F_{s}}$ are bulk sources; $\vartheta_{i}^{s}=\overline{s u_{i}}-\overline{u_{i}} \bar{s}$ are parameterizable subgrid fluxes.

The system of equations [4, 5, is solved by an explicit method. The conservative fourthorder accurate spatial approximation [18] and the second-order Adams-Bashforth scheme in time are applied. The Poisson equation is solved iteratively by the preconditioned conjugate gradient method. The equations are discretized on a regular staggered grid.

\subsection{Turbulent Closure}

A mixed subgrid/subfilter model [1] is used to compute the tensor $\tau_{i j}$ :

$$
\tau_{i j}^{m i x}=\tau_{i j}^{s m a g}+\tau_{i j}^{s s m}=-2\left(C_{s} \bar{\Delta}\right)^{2}|\bar{S}| \bar{S}_{i j}+\left(\overline{\bar{u}_{i} \bar{u}_{j}}-\overline{\overline{u_{i}}} \overline{\overline{u_{j}}}\right),
$$

where $\bar{S}_{i j}$ is the filtered strain-rate tensor; $C_{s}$ is the dimensionless coefficient variable in space and time dependent on the local flow characteristics and determined dynamically [6]. The details of the localized dynamic model and features of its numerical implementation are described in $[7]$. 
The turbulent diffusion model is used as a closure for scalar fluxes:

$$
\vartheta_{i}^{s}=-K_{h}{ }^{s u b g r} \frac{\partial \bar{s}}{\partial x_{i}}
$$

where $K_{h}^{\text {subgr }}=\left(1 / S c^{\text {subgr }}\right)\left(C_{s} \bar{\Delta}\right)^{2}|\bar{S}|$ is subgrid diffusivity coefficient. The turbulent subgrid Schmidt number $S c^{\text {subgr }}$ has a fixed value $S c^{\text {subgr }}=0.8$.

\subsection{Boundary Conditions}

In the numerical experiments presented below, a simple configuration of roughness elements is considered, which does not require accurate reproduction of the positions of flow separation and the formation of internal boundary layers. It is assumed that within the grid cell closest to the surface, the flow has a plane-parallel structure, which obeys the general laws of turbulent near-wall flow over the surface with small roughness elements.

The natural boundary conditions for the resolved velocity components at the solid boundaries of the region for staggered grids are the free slip and non-penetration:

$$
\partial \overline{\mathbf{u}}_{s} /\left.\partial n\right|_{\Gamma}=0 ;\left.\quad \bar{u}_{n}\right|_{\Gamma}=0
$$

where $\overline{\mathbf{u}}_{s}$ is the tangential velocity to the boundary $\Gamma$, and $\bar{u}_{n}$ is the normal velocity component. This conditions allows us to obtain an approximation of the convective terms of the equation of motion [4, which preserves the kinetic energy in the absence of viscosity.

The terms associated with the subgrid turbulent stresses are approximated in flux form, so that the wall friction is taken into account by setting the surface tangential stress vector $\tau_{s}=\left.\left(\tau_{i n} \tau_{j n}\right)\right|_{\Gamma}$, depending on the velocity $\overline{\mathbf{u}}_{s}=\left(u_{i}, u_{j}\right)$ at grid nodes distant from the boundary by a distance $\Delta_{g n} / 2\left(\Delta_{g n}\right.$ is the grid step in the direction of the normal $n$ to the surface):

$$
\left.\tau_{i n}\right|_{\Gamma}=-C_{u}^{2}\left|\mathbf{u}_{s}\right| \bar{u}_{i}
$$

The momentum exchange coefficient $C_{u}$ in these calculations was set the same for all surfaces of urban canopy (ground, roofs and walls) and corresponds to the value of the roughness parameter $z_{0} / h=6.25 \cdot 10^{-4}$, where $h$ is the height of objects.

Periodic boundary conditions are used at the lateral boundaries of the computational domain, and the condition 8 is used at the upper boundary. The boundary conditions for scalar quantities are similar to those for the velocity components tangential to the surface 8]. For the scalar transport, the source at the surface is specified as an additional constant flux $Q_{s}$.

\subsection{Parallel Implementation}

Algorithms for solving system of equations [4, 5, of the LES model are parallelized using MPI library. A three-dimensional decomposition of the computational domain is applied. At each model step and at each iteration in procedures for Poisson and dynamic Smagorinsky closure, the MPI-processes exchange with each other by data related to the boundary nodes of the decomposition subdomains. Collective communication calls are used to calculate the norms of vectors used in iterative methods and for spatial averaging in statistical data processing procedures. Depending on the spatial approximation of a particular differential operator, the data for exchanges are shifted by one or two grid nodes from the subdomain boundaries. Most of the exchanges between the processes are implemented using MPI non-blocking subroutines. 


\section{RANS Model Description}

For a horizontally homogeneous, unidirectional, neutrally stratified flow, the onedimensional (in the vertical direction) equations of the RANS model are as follows:

$$
\begin{aligned}
& \frac{\partial U}{\partial t}+\frac{\partial \tau}{\partial z}=-C_{d}|U| U+F_{e} \\
& \frac{\partial S}{\partial t}+\frac{\partial Q_{s}}{\partial z}=F_{s} .
\end{aligned}
$$

Here $U$, and $S$ are mean wind speed and scalar concentration, respectively. $F_{D}=-C_{d}|U| U$ is the quadratic form drag.

The turbulent momentum flux $\tau$ and turbulent kinematic scalar concentration flux $Q_{s}$ are calculated using the gradient approximation:

$$
\begin{gathered}
\tau=-K_{m} \frac{\partial U}{\partial z} \\
Q_{s}=-K_{h} \frac{\partial S}{\partial z},
\end{gathered}
$$

where $K_{m}$ and $K_{h}$ are the turbulent viscosity and diffusivity coefficients, which, in contrast to the LES formulation, do not depend on the length scale related to the filter width or the grid resolution. The system of equations (10, 11) corresponds to the simplified boundary layer model used in large-scale atmospheric models. We will consider a closure in which the coefficients $K_{m}$ and $K_{h}$ are determined from the similarity relations:

$$
\begin{aligned}
& K_{m}=S_{m} \frac{E_{k}^{2}}{\varepsilon}, \\
& K_{h}=S_{h} \frac{E_{k}^{2}}{\varepsilon} \equiv K_{m} / S c_{t} .
\end{aligned}
$$

The TKE is described by a prognostic equation:

$$
\frac{\partial E_{k}}{\partial t}-\frac{\partial}{\partial z} \frac{K_{m}}{\sigma_{k}} \frac{\partial E_{k}}{\partial z}=P+P_{D}-\varepsilon
$$

where $P_{D}$ is the TKE production through interactions with buildings, given by the formula 2, and $P$ is the TKE production by mean shear:

$$
P=K_{m}\left(\frac{\partial U}{\partial z}\right)^{2}
$$

The TKE dissipation rate $\varepsilon$ is defined with a diagnostic turbulent length scale $l_{\varepsilon}$ :

$$
\varepsilon=\frac{E_{k}^{3 / 2}}{l_{\varepsilon}}
$$

In the equations 12 15 $S_{m}$ and $S_{h}$ are universal functions determining, in particular, the turbulent Schmidt number $S c_{t}=S_{m} / S_{h}, \sigma_{k}$ is the closure parameter responsible for the TKE diffusion. Here, we will follow the traditional approach and assume $\sigma_{k}=1$. In stratified turbulent flows, the functions $S_{m}$ and $S_{h}$ depend on the Richardson number $R i$. Since in these calculations buoyancy was not considered, we set constant values: $S_{m}=0.09, S c_{t}=0.8, S_{h}=S_{m} / S c_{t}$. 
Obviously, the interactions between flow and buildings significantly changes the scale of turbulent vortices both inside and above the urban canopy. In the standard model described above, a single length scale $l_{\varepsilon}$ is introduced, and all other length scales are proportional to it, since the model coefficients are fixed and do not change values depending on the solution and position in space. The standard choice for the turbulent scale over a flat wall is:

$$
l_{\varepsilon}=l_{T}=\kappa z
$$

In the external flow over the urban medium (at $z>h$ ) we can use approximation:

$$
l_{T}=\kappa\left(z-D_{u}\right)
$$

It remains to choose the length scale in the layer $0<z<h$. A separate section 5 of this paper is devoted to this issue, where we will discuss construction of the length scale using methods reminiscent of those of similarity theory. Thus, we retain the standard and well-known K-l model of turbulence (see, for example, 2]) without changing the relations between length scales and without introducing any other corrections responsible for time scales.

An alternative approach is to introduce unknown, different functions $\psi_{i}$ of arbitrary form within layer $0<z<h$ for length scales of dissipation $l_{\varepsilon}$, velocity $l_{m}$, scalar fluctuations, and so on, which are assumed universal: $l_{\varepsilon}=z \psi_{\varepsilon}\left(z / h, \varsigma_{1}, \varsigma_{2}, \varsigma_{3}, \ldots.\right), l_{m}=z \psi_{m}\left(z / h, \varsigma_{1}, \varsigma_{2}, \varsigma_{3}, \ldots\right), \ldots$. Here, $\varsigma_{1}, \varsigma_{2}, \varsigma_{3}, \ldots$ is a set of dimensionless parameters generated from all geometric dimensions of the underlying surface. In fact, this is the approach proposed in [19] to construct $l_{\varepsilon}$ and $l_{m}$, where the functions $\psi_{\varepsilon}$ and $\psi_{m}$ are determined and calibrated from LES data. The main defect of this method is the difficulty of determining a limited set of leading dimensionless parameters $\varsigma_{i}, i<N \sim 1$. Moreover, this choice may not be universal with respect to the underlying surface geometry.

We do not cite formulas from 19 in this paper due to their unwieldiness and some differences in writing in terms of the system of equations 12$]-15$. However, we have adopted their turbulence model within our RANS code and will compare its results with those obtained using the $l_{T}$ scale described below.

\section{Numerical Experiments Design}

\subsection{LES Model}

Here we present the results of three simulations performed on an equilateral grid of $512 \times 256 \times 128$ nodes. The experiments EXP1, EXP2, and EXP3 (see, Fig. 1 19) differ in the geometry and location of the objects on the lower boundary of the area (cubes and rectangular parallelepipeds simulating buildings). In this figure: $h$ and $h / 2$ are the heights of the objects (there are 32 grid steps $\Delta$ per height $h$ ). The height $h$ will further be used as a length unit to normalize the results. The size of the entire computational domain was: $L_{x} \times L_{y} \times L_{z}=16 h \times 8 h \times 4 h$.

The flow is accelerated by a constant external pressure gradient $F_{i}^{e}=-d P / d x=U_{* f i x}^{2} /\left(L_{z}-h\right)$, where $U_{* f i x}$ is the given value of friction velocity at height $h$ in equilibrium. The initial profiles of the mean streamwise velocity at $z>D_{u}$ were calculated using equation [3] and from preliminary estimates of the parameters $z_{0 u}$ and $D_{u}$. The average scalar concentration was set equal to zero in the entire computational domain. An additional term is added to the right-hand side of the prognostic equation of the scalar 
(a)
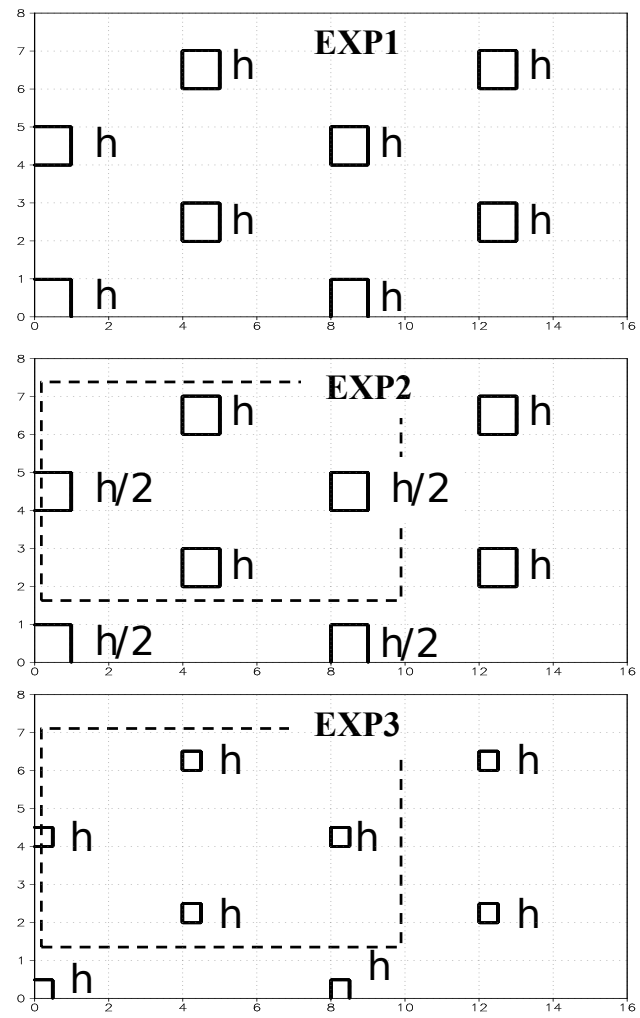

(b)
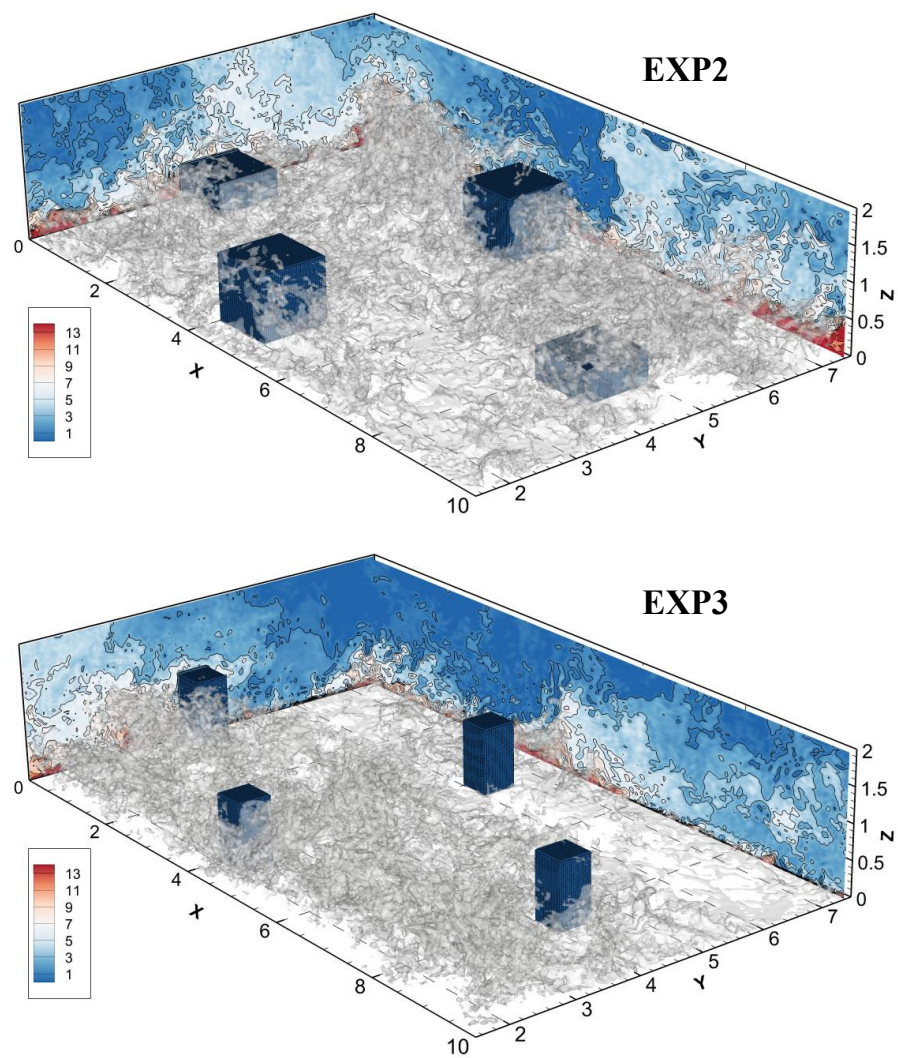

Figure 1. (a) configuration of streamlined objects; (b) visualization of the instantaneous flow fields in EXP2 and EXP3 (inside the area denoted by the dotted line on the left); the color on the section shows the normalized scalar concentration transported by the turbulent flow $\left(S-S_{\text {top }}\right) / S_{*} ;$ isosurface $-\left(S-S_{\text {top }}\right) / S_{*}=7$

concentration $\bar{s}$ to compensate for the trend of the mean concentration, which is independent of the coordinates:

$$
\overline{F_{s}}=\frac{1}{V_{\text {air }}} \int_{\Gamma}-Q_{s} d \Gamma
$$

where $V_{\text {air }}$ is the volume of the part of the model domain filled with air, and $\Gamma$ is the surface on which the concentration flux $Q_{s}$ is set (in this case, the "ground" surface).

To initialize the simulations, random noise of small amplitude was imposed on the initial conditions. The calculations were run for 40 units of dimensionless time $\tilde{t}=L_{z} / U_{* \text { fix }}$, the last 10 units of dimensionless time were used to average the results. To normalize the scalar concentration profiles, the presented results use the turbulent concentration scale $S_{*}=\left\langle w^{\prime} s^{\prime}\right\rangle_{h} / U_{*}$, where $\left\langle w^{\prime} s^{\prime}\right\rangle_{h}$ is the total time- and space-averaged steady-state scalar flux (including subgrid diffusion) at height $z=h+1.5 \Delta$ ( $\Delta$ is the model grid step). The velocity is normalized by the friction velocity $U_{*}$ at the same height. Since we set the scalar flux from the surface rather than calculating it, we compare the LES results with the RANS results by the scalar concentration defect $\left(S-S_{\text {top }}\right) / S_{*}$, where $S_{\text {top }}$ is the calculated concentration near the top of the model domain. From the physical point of view, this means that we compare the concentrations calculated in LES and RANS under the condition of equality of their fluxes from the ground surface and that at the height $L_{z}$ is scalar concentration is equal to zero. Figure 1 shows the concentration 
Turbulent Length Scale for Multilayer RANS Model of Urban Canopy and Its...

$\left(S-S_{\text {top }}\right) / S_{*}$ (one of the instantaneous states) in experiments EXP2 and EXP3 to demonstrate the turbulent nature of the flow.

\subsection{RANS-models}

The conditions of numerical experiments with one-dimensional RANS models are identical to those in LES. The same height of the computational region $L_{z}=4 h$ and the same roughness parameter of the ground surface $z_{0} / h=6.25 E-4$ were chosen. The grid in all RANS-model experiments matched the vertical grid of the LES model. For correctness of comparison here we will not apply any parameterizations of the drag coefficient $C_{D}(z)$ in both models, but will take its "true" value obtained from LES results (see section 4. Additional checks have shown that, at qualitative level, the conclusions presented below do not change when the parameterized coefficient $C_{D}$ is substituted.

\section{LES Results}
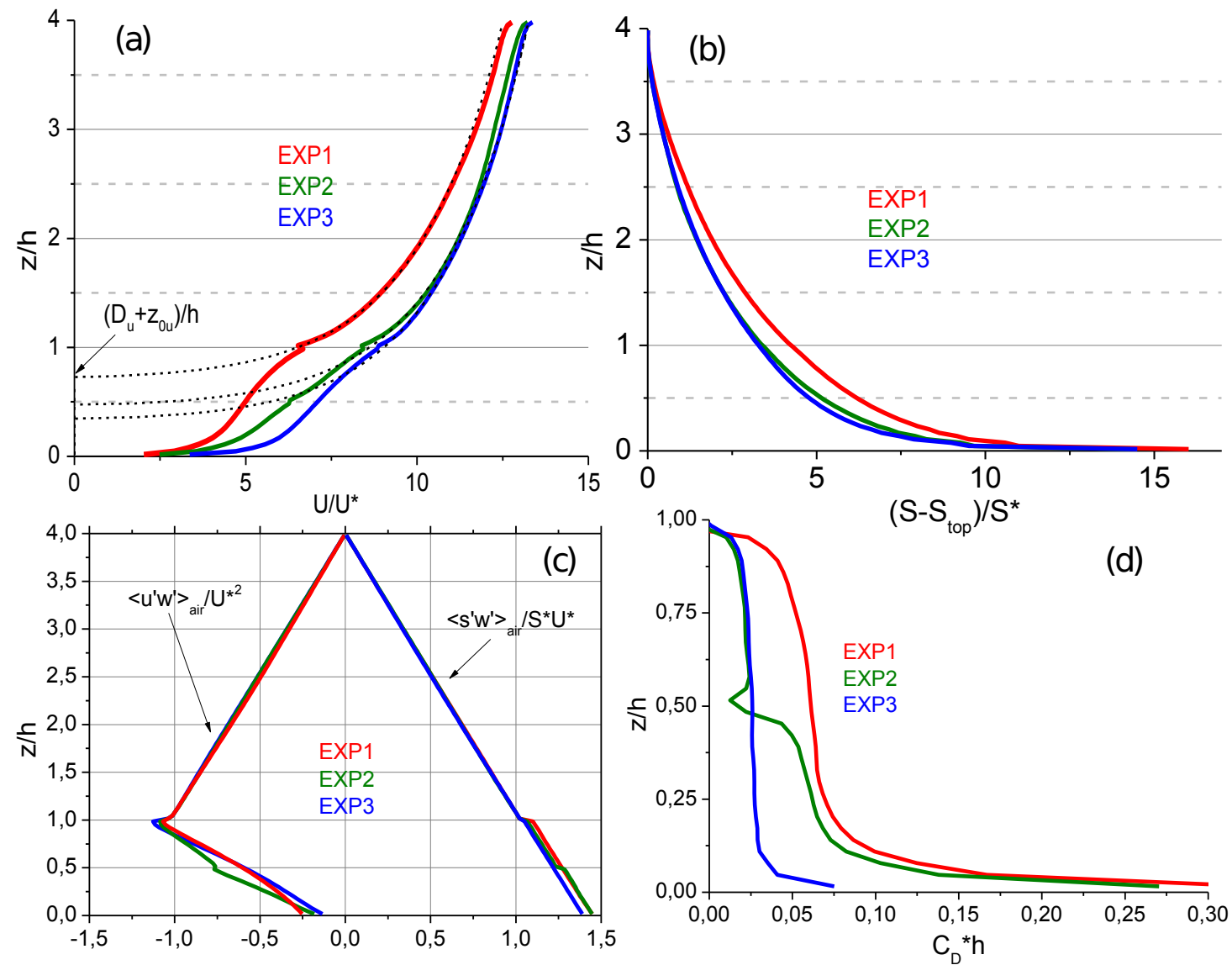

Figure 2. Results of LES-model experiments with configurations EXP1, EXP2 and EXP3: (a) normalized velocity $U / U *$, (b) scalar concentration defect $\left(S-S_{t o p}\right) / S_{*}$, (c) normalized momentum and concentration fluxes (averaging over part of the region occupied by air), (d) normalized form drag coefficient $h C_{D}$

Some results of the LES numerical experiments are shown in Fig. 2, Here Fig. 2a shows the average flow velocity in all three experiments. The dotted curves approximate the velocity 
profiles by logarithmic law 3 . Displacement heights $D_{u}$ and roughness parameters $z_{0 u}$ were determined by minimizing the standard deviation of the approximations from the LES profiles at the model grid cell located in the height interval $1.1<z / h<2.6$. Figure 2 a shows that the dependence 3 sufficiently well approximate the simulated mean velocity. The largest deviations of the approximation from the values obtained in LES are observed for the surface in EXP2 with variable roughness elements height. In addition, Fig. 2 shows that the surfaces EXP2 and EXP3 produce approximately the same aerodynamic drag for the external turbulent flow above them, since the average flow velocities in these calculations are close to each other. The surface EXP1 has greater aerodynamic drag and provides a lower flow velocity for the same external force accelerating the flow. The data on the values of parameters $D_{u}$ and $z_{0 u}$ are presented in Tab. 1 . The dimensionless scalar concentration defects are shown in Fig. 2p. This figure shows that the

Table 1. Roughness length $z_{0 u}$

and displacement height $D_{u}$

determined from the mean

average velocity profiles for

different surface configurations

\begin{tabular}{ccl}
\hline & $z_{0 u} / h$ & $D_{u} / h$ \\
\hline$E X P 1$ & $2.2 \cdot 10^{-2}$ & 0.7 \\
\hline$E X P 2$ & $1.76 \cdot 10^{-2}$ & 0.45 \\
\hline$E X P 3$ & $1.83 \cdot 10^{-2}$ & 0.32 \\
\hline
\end{tabular}

surfaces EXP2 and EXP3 provide approximately the same ventilation of the layer $0<z / h<0$, and for the surface EXP1 the concentration of the impurity in the lower part is higher.

Figure 24 shows the full (including the explicitly resolved and subgrid part in LES) scalar and momentum fluxes, normalized to the corresponding turbulent scales. It can be seen from Fig. 24 that in all three experiments an equilibrium state is reached, in which the divergence of the mean fluxes balances a constant external force: for both concentration and momentum, the fluxes have a linear form at $z / h>1$. Here, the averaging was performed only over the part of the computational domain occupied by air, so the concentration fluxes in Fig. 2, have discontinuities at heights where the area occupied by the objects changes. When averaged over the entire computational domain and considering zero fluxes inside the objects, the heat fluxes are continuous and piecewise linear. The momentum flux profiles in this averaging will have discontinuities due to subgrid friction against the top boundary of the objects and due to the abrupt change in form drag.

The normalized drag coefficient $C_{D} h$ obtained for the different LES experiments is shown in Fig. 2p. This coefficient is calculated from the balance of mean forces in the equilibrium state:

$$
C_{D} U_{a i r}^{2}=-\frac{\partial P}{\partial x}-\frac{\partial\left\langle u^{\prime} w^{\prime}\right\rangle_{a i r}}{\partial z} .
$$

Here, the averaging is also performed over the part of the region filled with air. The corresponding correction factor $S_{a i r} / S_{t o t}$ differing slightly from unity is taken into account when substituting the factor $C_{D}(z)$ in the RANS model, where no real objects are present. Note that EXP2 and EXP3 produce the same coefficient $C_{D}$ at the top of the canopy layer. This is apparently due to the fact that the frontal area of objects per unit volume in these calculations at $0.5<z / h<1$ is the same, and the interposition of objects does not have much influence on the value of $C_{D}$ 
at their rather sparse distribution. Note that although EXP2 and EXP3 configurations differ significantly in the value of $C_{D}$ at small values of $z / h$, they set approximately the same resistance for the external flow (the mean velocities of the external flows in EXP2 and EXP3 are close to each other), that is: for the value of $z_{0 u}$ the upper part of the urban geometry has a determining influence. As it will be seen from the results presented in section 6, none of the multilayer RANS models tested reproduce this effect.

\section{Turbulent Length Scale for RANS}

(a)

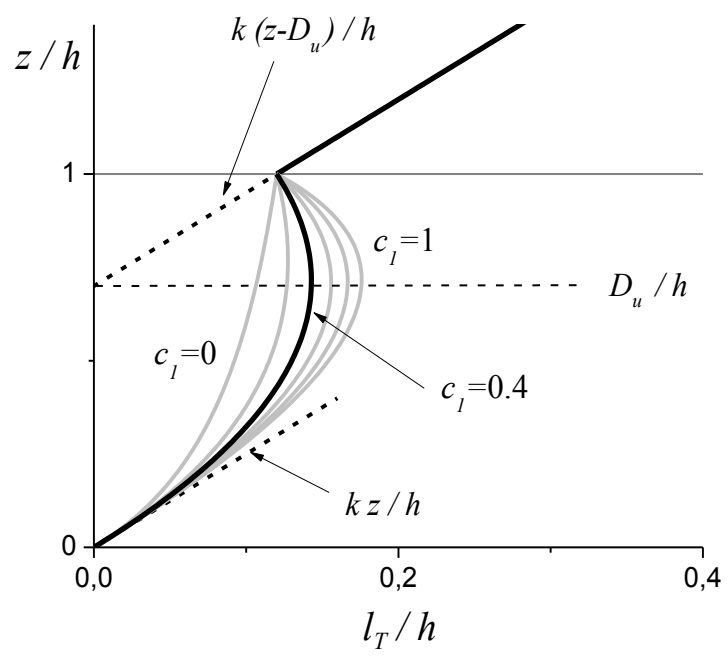

(b)

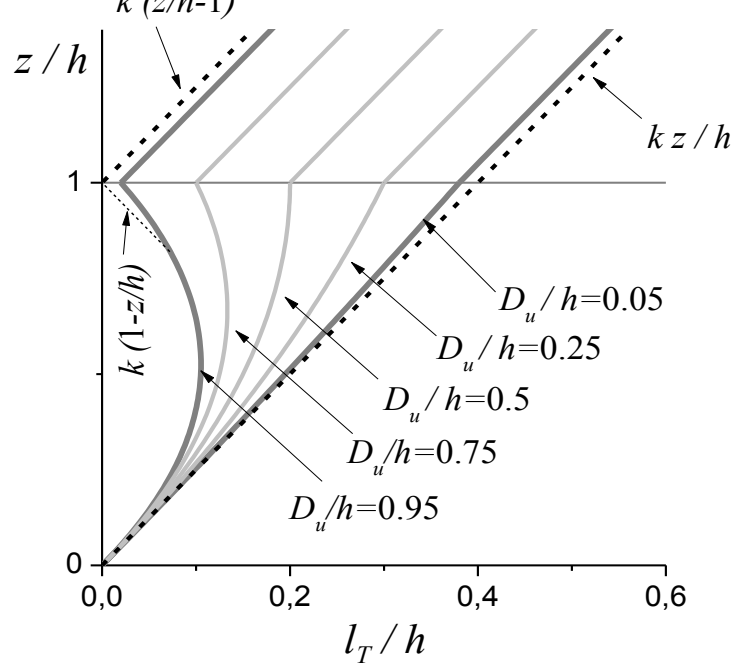

Figure 3. (a) variations of the turbulent scale $l_{T} 23$ with changes in $c_{1}$ (grey solid lines). The curve at $c_{1}=0.4$ is highlighted in black. All simulations with the RANS model shown in Fig. 4 are performed with this value of $c_{1}$. (b) variations of $l_{T}$ with changes in $D_{u}$ (here, $c_{1}=0.4$ )

As noted in the introduction, the length scale $D_{u}$ (along with some average building height scale $\langle h\rangle$ and ground surface distance $z$ ) is one of the defining turbulent length scales, as indicated by the calculated velocity profiles (see Fig. 2 that are close to logarithmic dependence 3 . Indeed, when the velocity gradient is normalized by length scale $z^{\prime}=z-D_{u}$ we obtain a value close to the constant:

$$
\frac{d U}{d z} \frac{z-D_{u}}{U_{*}} \approx \frac{1}{\kappa}
$$

from which the expression 3 follows. In turn, this means that the scale $z^{\prime}=z-D_{u}$ characterizes the spatial spectra and co-spectra of turbulent fluctuations above urban canopy (see spectral analysis of such turbulent flows in papers [9] and [10]). Since there is no clear boundary between the urban canopy and the atmosphere above it, we are entitled to assume that the spectral characteristics of turbulence in these two layers under the influence of disturbances from streamlined objects change in a such way, that one can establish the similarity between them using length scales $D_{u},\langle h\rangle$ and $z$. In this case, $D_{u}$ acts as a length scale that has already absorbed all the geometric parameters of the urban environment. The simplest, and in some cases successful, technique to obtain one generalized length scale from several is inverse interpolation of scales with some weighting coefficients. This is equivalent to averaging with the weights of the corresponding wave numbers and it approximates, for example, the co-spectrum-weighted 
average wave numbers proportional to the inverse scale of the Prandtl mixing length, which, in fact, we need to calculate the turbulent viscosity and diffusion coefficients (see [9]). Since the RANS model with only TKE prognostic equation in its most simplified form needs only one scale of length $l_{T}$, and all others are considered proportional to it, we will use the following approximation:

$$
\frac{1}{l_{T}}=\frac{1}{c_{z} z}+\frac{1}{c_{D} D_{u}},
$$

where $c_{z}$ and $c_{D}$ are undefined functions depending on two dimensionless quantities $z /\langle h\rangle$ and $\langle h\rangle / D_{u}$. We can impose the following constraints these functions need to satisfy:

$$
l_{T} \rightarrow \kappa z, \quad \text { for } z \rightarrow 0
$$

and

$$
l_{T}=\kappa\left(\langle h\rangle-D_{u}\right), \quad \text { for } z=\langle h\rangle .
$$

The constraint 21 means that the length scale we obtain should transition into the usual near-wall turbulence scale $\kappa z$ when approaching the ground surface, and the constraint 22 ensures continuity of the length scale as we transition from the canopy layer to the external flow above it.

Conditions 21] and 22 allow us to set $c_{z}=\kappa$, and also impose a restriction on the type of function $c_{D}=c_{D}\left(z /\langle h\rangle,\langle h\rangle / D_{u}\right)$. To calculate $l_{T}$ we use the following linear with respect to $z^{\prime}=(\langle h\rangle-z) / D_{u}$ expression for $c_{D}$ :

$$
c_{D}=\kappa\left[\left(\frac{\langle h\rangle}{D_{u}}\right)^{2}-\frac{\langle h\rangle}{D_{u}}\right]+c_{1} z^{\prime} f_{1}\left(\frac{\langle h\rangle}{D_{u}}\right),
$$

where $c_{1}$ is a constant, $f_{1}$ is a function, which depends on only one variable $\langle h\rangle / D_{u}$. Note that in the expansion 23) the first term is determined by the constraint 22. Let us consider the case $f_{1} \equiv 1$, which allows us to obtain an expression for $l_{T}$ containing only one constant $c_{1}$. The dependence of the turbulent scale on the height $z /\langle h\rangle$ at different values of $c_{1}$ is shown in Fig. 3a. Figure $3 \mathrm{~b}$ shows the changes of turbulent scale $l_{T}$ with varying displacement height $D_{u}$ value. It can be seen that the scale $l_{T}$ approaches $\kappa z$ in the entire layer $0<z<h$ when $D_{u}$ goes to zero, i.e. in the case when the influence of the canopy is negligible.

The function 23. allows us to set a maximum in the value of $l_{T}$ at some height from the surface inside the canopy, $z<h$, and at that corresponds to a polynomial of small degree from the arguments $z /\langle h\rangle$ and $D_{u} /\langle h\rangle$.

The determination of additional parameters $c_{1}$ and, in the general case, of the function $f_{1}$ requires large set of LES experiments for different configurations of the roughness elements within urban canopy. Further, when setting $l_{T}$ and evaluating RANS model with LES data, for simplicity we will assume that $c_{1}=0.4$ (the curve is highlighted in black in Fig. 3 .

\section{RANS Results and Their Comparison with LES Data}

Here we compare only the velocity and scalar concentration profiles in the equilibrium state, since they are what the simplified RANS-models are supposed to reproduce. In addition, in authors opinion, comparing the TKE in one-dimensional RANS and the TKE in LES is incorrect, especially within the roughness layer, for a number of reasons, such as: the presence of passive large-scale structures in LES that do not carry momentum and concentration vertically; 
the presence of a two-dimensional dispersion component of the time-averaged horizontal velocity associated with the flow's envelopment of obstacles; and the substantial anisotropy of turbulent fluctuations, which is only implicitly accounted for this type of the flow in one dimensional RANS model. A comparison of the TKE in LES and the TKE in RANS would require an interpretation which deserves a separate study.
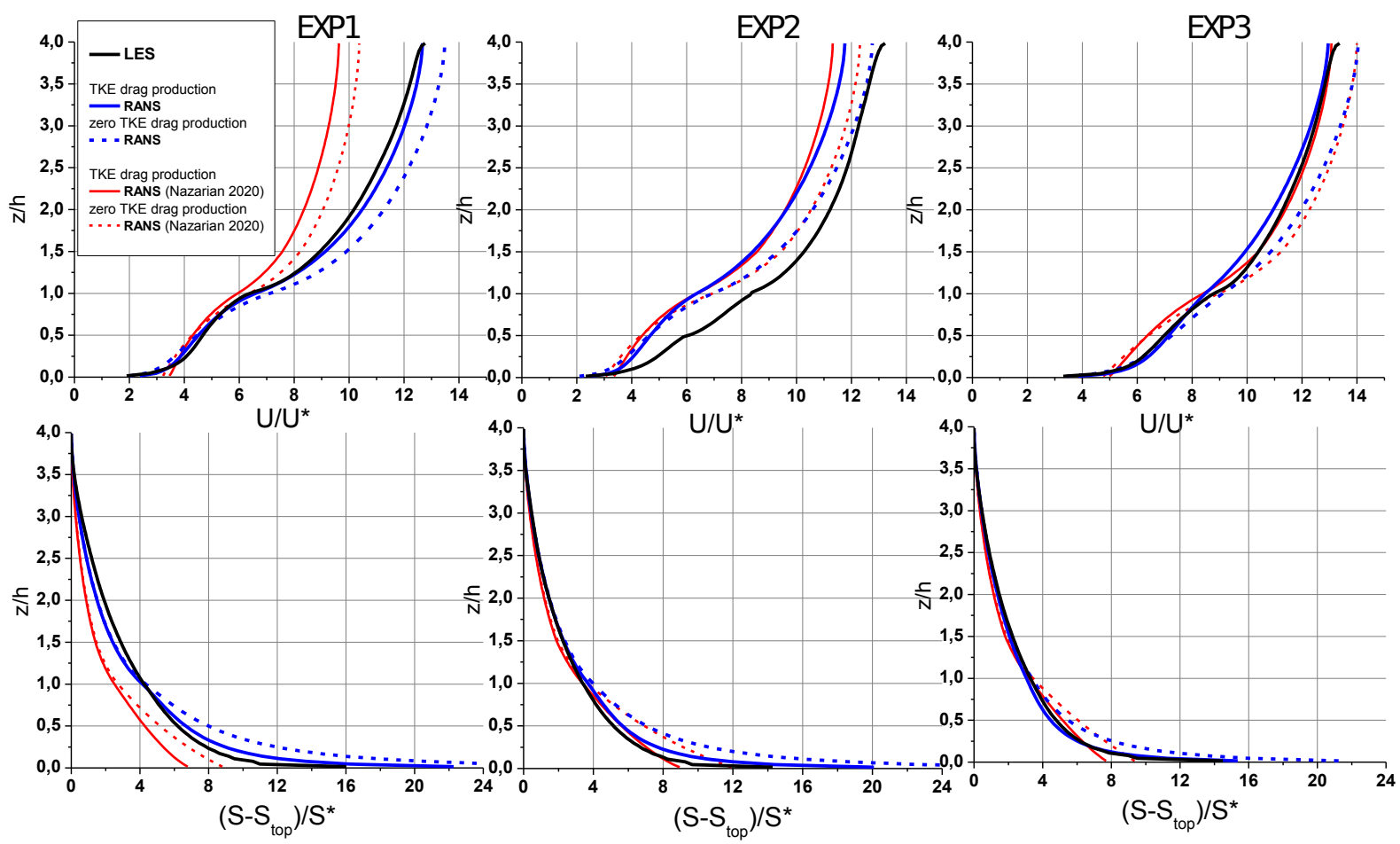

Figure 4. Comparison of LES and RANS results. The black curves are LES results. Blue curves profiles calculated using the turbulent length scale shown in Fig. 3. red curves - according to the turbulent length scale model from [19]. The solid lines are RANS with TKE production term due to form drag 22; the dashed lines correspond to the ones without this additional production term. Left, center and right columns correspond to EXP1, EXP2 and EXP3 configurations respectively

Figure 4 shows the results of calculations using the RANS models in comparison with the LES data (black curves). Profiles of the normalized velocity $U / U_{*}$ are plotted in the upper row, and the lower row corresponds to the normalized scalar concentration defect $\left(S-S_{t o p}\right) / S_{*}$. The blue curves show profiles calculated using the turbulent length scale described in section 5 , and the red curves show profiles obtained using the turbulent length scale model proposed in [19]. The solid lines show the results obtained using the full TKE balance model, which includes TKE production due to form drag 2), and the dashed curves show the results RANS simulations in which this term in the TKE balance equation 13 is omitted. The plots are arranged by columns, each representing different geometry configurations of urban canopy.

Let us highlight the most characteristic differences between the results obtained with the model proposed in this study and the model [19]. Near the ground surface, the velocity and scalar 
gradients reproduced by the proposed model increase towards the surface, which is a reflection of the correct asymptotic of the near-wall flow observed also in LES results. Inside the $0<z<h$ layer, the profiles calculated using the proposed $L_{T}$ scale have a regular curved shape, reflecting the absence of excess turbulent viscosity and diffusion. In most cases (except for the velocity profile in EXP2), adding extra TKE production associated with turbulent flow interactions with buildings improves simulation results. In the 19] model, all profiles appeared excessively mixed within the $0<z<h$ layer, and no near-ground asymptotic are observed. These exact features can also be seen in the authors original paper (see mean velocity in Figs. 12 and 14 from [19]). Note that here we have made more rigid requirements to RANS models by using a low density of the buildings configurations (plane density for the EXP1 is equal to $6.25 \cdot 10^{-2}$ - lower limit of what was modeled in [19], and EXP2 and EXP3 has even lower plane density), so that the defects of turbulent closure in reproducing velocity profiles are more appreciable than the influence of form drag. Second, we have considered transport of scalars for which this volume force is absent. The manifestation of asymptotic of the logarithmic layer near the surface in RANS simulations allows us to hope that with such a model it is possible to refine modelling of heat and moisture exchange with the ground surface in urban canopies, for which Monin-Obukhov similarity theory is of most importance.

Both RANS models equally incorrectly reproduced the velocity profile in EXP2. The profiles obtained in RANS are shifted relative to the LES profile to the left, that is, the canopy modelled by RANS creates a greater aerodynamic resistance to external flow than the explicitly resolved canopy in LES experiments. In terms of approximations 3, this means that the roughness parameter $z_{0 u}$ of the model surface was overestimated. In section 4 , we noted that, judging from the comparison of EXP2 and EXP3 results, the upper part of the objects is more responsible for the efficiency of momentum exchange between the external flow and the surface as a whole. We do not have a ready recipe for taking this effect into account in the RANS models, but we hope that this problem can also be solved by involving dimensional considerations.

\section{Conclusions}

In this paper, we performed numerical experiments with LES model of neutrally stratified flow over an urban-type surface with a low density of roughness elements. The LES was used as a benchmark for evaluation of multilayer local one-dimensional RANS models of urban canopy. Due to the development of supercomputer technology, such models may, in the near future, become units of weather prediction and climate atmospheric models and will allow detailing weather forecasts and assessing possible risks during climatic changes in urban environments of specific megapolises.

In order to construct a one-dimensional RANS model of the urban canopy, we deliberately chose the simplest possible approach, being limited to the standard K-l turbulence closure and did not adjust its constants for specific type of the flow. However, the choice of simple turbulent length scale, which logically follows from the dimensional analysis and trivial considerations about the spectral structure of urban canopy turbulence, gives reassuring results. The main "null hypothesis" was the assumption that the wide set of key length scales necessary to generalize the geometric characteristics of the urban environment is already contained in the spectral structure of the turbulent flow over the urban canopy. The main integral and "measurable" parameter, which reflects the impact of the surface geometry as a whole on the spatial spectra, is the displacement height $D_{u}$. Based on this hypothesis, we proposed a parameterization of 
the turbulent length scale within the urban canopy, which includes $D_{u}$ as one of the main dimensional parameters, and does not incorporate the analysis of the geometry of objects as such.

The proposed turbulent length scale parameterization allows simple tuning of RANS models with just one constant without losing physically valid asymptotics near the ground surface and near the upper boundary of the urban canopy. More rigorous validation and tuning of the proposed model requires consideration of a wide range of surface configurations, which will require additional supercomputer computational experiments with eddy-resolving turbulent flow models (LES and DNS).

Identified defects of the proposed closure are indicated in section 6. In particular, our model, as well as the model [19], was unable to correctly reproduce the velocity of the external flow for canopy with variable heights of roughness objects and overestimates the aerodynamic drag of such a surface as a whole. Additional LES calculations with surfaces of this type are needed to elucidate the reasons for this defect and to correct it. Here we have considered only neutrally stratified flows, however there is a greater interest for flows which are affected by buoyancy forces, characteristic for the atmospheric boundary layer, as well as - processes of heat and moisture exchange with the urban surface. Such flows over the urban canopy can also be studied with LES-models (see, for example, [10]). Comparison of the results of multilayer RANS models with the data of eddy-resolving simulations is the most straightforward way to evaluate and improve such turbulence closures.

\section{Acknowledgements}

Numerical experiments, their analysis and the development of new parameterization for RANS of urban layer (sections 3, 4 and 5 was performed with financial support of the Russian Science Foundation, grant 21-71-30023.

LES and RANS model code development and implementation of numerical algorithms for solving of LES and RANS equations (sections 1 and 2 was supported by INM RAS Division of Moscow Center for Fundamental and Applied Mathematics (agreement No. 075-15-2019-1624).

This paper is distributed under the terms of the Creative Commons Attribution-Non Commercial 3.0 License which permits non-commercial use, reproduction and distribution of the work without further permission provided the original work is properly cited.

\section{References}

1. Bardina, J., Ferziger, J., Reynolds, W.: Improved subgrid-scale models for large-eddy simulation. In: 13th fluid and plasmadynamics conference. p. 1357 (1980). https://doi.org/ $10.2514 / 6.1980-1357$

2. Burchard, H.: Applied turbulence modelling in marine waters, vol. 100. Springer Science \& Business Media (2002)

3. Cheng, H., Castro, I.P.: Near wall flow over urban-like roughness. Boundary-Layer Meteorology 104(2), 229-259 (2002). https://doi.org/10.1023/A:1016060103448

4. Coceal, O., Thomas, T., Castro, I., Belcher, S.: Mean flow and turbulence statistics over groups of urban-like cubical obstacles. Boundary-Layer Meteorology 121(3), 491-519 (2006). 
https://doi.org/10.1007/s10546-006-9076-2

5. Dupont, S., Otte, T.L., Ching, J.K.: Simulation of meteorological fields within and above urban and rural canopies with a mesoscale model. Boundary-Layer Meteorology 113(1), 111-158 (2004). https://doi.org/10.1023/B:BOUN.0000037327.19159.ac

6. Germano, M., Piomelli, U., Moin, P., Cabot, W.H.: A dynamic subgrid-scale eddy viscosity model. Physics of Fluids A: Fluid Dynamics 3(7), 1760-1765 (1991). https://doi.org/10. $1063 / 1.857955$

7. Glazunov, A., Rannik, Ü., Stepanenko, V., et al.: Large-eddy simulation and stochastic modeling of lagrangian particles for footprint determination in the stable boundary layer. Geoscientific Model Development 9(9), 2925-2949 (2016). https://doi.org/10. 5194/gmd-9-2925-2016

8. Glazunov, A.V.: Numerical simulation of turbulence and transport of fine particulate impurities in street canyons. Numerical methods and programming 19, 17-37 (2018)

9. Glazunov, A.: Numerical modeling of turbulent flows over an urban-type surface: Computations for neutral stratification. Izvestiya, Atmospheric and Oceanic Physics 50(2), 134-142 (2014). https://doi.org/10.1134/S0001433814010034

10. Glazunov, A.: Numerical simulation of stably stratified turbulent flows over an urban surface: Spectra and scales and parameterization of temperature and wind-velocity profiles. Izvestiya, Atmospheric and Oceanic Physics 50(4), 356-368 (2014). https://doi.org/10. $1134 / \mathrm{S} 0001433814040148$

11. Glazunov, A.: Numerical simulation of stably stratified turbulent flows over flat and urban surfaces. Izvestiya, Atmospheric and Oceanic Physics 50(3), 236-245 (2014). https://doi. org/10.1134/S0001433814030037

12. Kanda, M., Kanega, M., Kawai, T., et al.: Roughness lengths for momentum and heat derived from outdoor urban scale models. Journal of Applied Meteorology and Climatology 46(7), 1067-1079 (2007). https://doi.org/10.1175/JAM2500.1

13. Kanda, M., Kawai, T., Moriwaki, R., et al.: Comprehensive outdoor scale model experiments for urban climate (COSMO). In: Proc., 6th Int. Conf. on Urban Climate. pp. 270-273 (2006). https://doi.org/10.1023/A:1016060103448

14. Krayenhoff, E.S., Jiang, T., Christen, A., et al.: A multi-layer urban canopy meteorological model with trees (BEP-Tree): Street tree impacts on pedestrian-level climate. Urban Climate 32, 100590 (2020). https://doi.org/10.1016/j.uclim.2020.100590

15. Krayenhoff, E., Christen, A., Martilli, A., Oke, T.: A multi-layer radiation model for urban neighbourhoods with trees. Boundary-layer meteorology 151(1), 139-178 (2014). https: //doi.org/10.1007/s10546-013-9883-1

16. Krayenhoff, E., Santiago, J.L., Martilli, A., et al.: Parametrization of drag and turbulence for urban neighbourhoods with trees. Boundary-Layer Meteorology 156(2), 157-189 (2015). https://doi.org/10.1007/s10546-015-0028-6 
17. Martilli, A., Clappier, A., Rotach, M.W.: An urban surface exchange parameterisation for mesoscale models. Boundary-layer meteorology 104(2), 261-304 (2002). https://doi.org/ 10.1023/A:1016099921195

18. Morinishi, Y., Lund, T., Vasilyev, O., Moin, P.: Fully conservative higher order finite difference schemes for incompressible flows. J. Comp. Phys. 143, 90-124 (1998). https: //doi.org/10.1006/jcph.1998.5962

19. Nazarian, N., Krayenhoff, E.S., Martilli, A.: A one-dimensional model of turbulent flow through "urban" canopies (MLUCM v2.0): updates based on large-eddy simulation. Geoscientific Model Development 13(3), 937-953 (2020). https://doi.org/10.5194/ gmd-13-937-2020

20. Santiago, J., Martilli, A.: A dynamic urban canopy parameterization for mesoscale models based on computational fluid dynamics Reynolds-averaged Navier-Stokes microscale simulations. Boundary-layer meteorology 137(3), 417-439 (2010). https://doi.org/10.1007/ s10546-010-9538-4

21. Xie, Z., Castro, I.P.: LES and RANS for turbulent flow over arrays of wall-mounted obstacles. Flow, Turbulence and Combustion 76(3), 291-312 (2006). https://doi.org/10. $1007 /$ s10494-006-9018-6 